\title{
Is chest X-ray screening for lung cancer in smokers cost-effective? Evidence from a population-based study in Italy
}

\author{
Paolo Pertile ${ }^{1 *}$, Albino Poli ${ }^{2}$, Lorenzo Dominioni ${ }^{3}$, Nicola Rotolo ${ }^{3}$, Elisa Nardecchia ${ }^{3}$, Massimo Castiglioni ${ }^{3}$, \\ Massimo Paolucci ${ }^{4}$, William Mantovani ${ }^{2,5}$ and Andrea Imperatori ${ }^{3}$
}

\begin{abstract}
Background: After implementation of the PREDICA annual chest X-ray (CXR) screening program in smokers in the general practice setting of Varese-Italy a significant reduction in lung cancer-specific mortality (18\%) was observed. The objective of this study covering July 1997 through December 2006 was to estimate the cost-effectiveness of this intervention.

Methods: We examined detailed information on lung cancer (LC) cases that occurred among smokers invited to be screened in the PREDICA study (Invitation-to-screening Group, $n=5815$ subjects) to estimate costs and qualityadjusted life-years (QALYS) from LC diagnosis until death. The control group consisted of 156 screening-eligible smokers from the same area, uninvited and unscreened, who developed LC and were treated by usual care. We calculated the incremental net monetary benefit (INMB) by comparing LC management in screening participants $(n=1244$ subjects) and in the Invitation-to-screening group versus control group.

Results: The average number of QALYS since LC diagnosis was 1.7, 1.49 and 1.07, respectively, in screening participants, the invitation-to-screening group, and the control group. The average total cost (screening + management) per LC case was higher in screening participants $(€ 17,516)$ and the Invitation-to-screening Group $(€ 16,167)$ than in the control group $(€ 15,503)$. Assuming a maximum willingness to pay of $€ 30,000 / Q A L Y$, we found that the intervention was cost-effective with high probability: $79 \%$ for screening participation (screening participants vs. control group) and $95 \%$ for invitation-to-screening (invitation-to-screening group vs. control group).
\end{abstract}

Conclusions: Based on the PREDICA study, annual CXR screening of high-risk smokers in a general practice setting has high probability of being cost-effective with a maximum willingness to pay of $€ 30,000 / Q A L Y$.

Keywords: Lung cancer, Chest X-ray screening, Cost-effectiveness, Cost-utility

\section{Background}

Lung cancer (LC) is the most deadly malignancy, with an estimated 1,200,000 deaths each year worldwide [1]. More than $85 \%$ of LC deaths are attributable to smoking cigarettes; therefore, tobacco control and smoking cessation are the interventions most commonly used to reduce LC morbidity and mortality $[1,2]$. Because heavy smokers

\footnotetext{
${ }^{*}$ Correspondence: paolo.pertile@univr.it

${ }^{1}$ Department of Economics, University of Verona, Via dell'Artigliere 19,

37129 Verona, Italy

Full list of author information is available at the end of the article
}

remain at high risk for many years, even after quitting [3], screening for LC is another intervention to consider. Screening aims to diagnose the disease at an early stage, when it is potentially curable with appropriate treatment protocols [4-7]. After lengthy methodological controversy over the efficacy of screening for LC, in recent years the results of observational screening with chest computed tomography (CT) in heavy smokers has shown a significant reduction of LC mortality [8]; with chest X-ray (CXR) screening the LC mortality reduction was less pronounced but still significant $[9,10]$. After publication in 2011 of the National Lung Screening Trial (NLST) 
results, which showed a $20 \%$ reduction in LC mortality with CT screening compared with CXR screening [11], major cancer organizations began to advocate for lowdose chest CT screening with the NLST criteria [12]. However, depending on the model and the assumptions applied, estimates of the cost-effectiveness of CT screening ranged widely, from $\$ 19,500$ (USD) [13] to $\$ 2,322,700$ [14] per quality-adjusted life-year (QALY) gained. The wide divergence of these estimates contributes to the current uncertainty about cost-effectiveness and affordability of CT screening in the general population setting [15-18]. While CT screening has been extensively analyzed, including its economic implications, the assessment of cost-effectiveness of CXR screening in smokers has received little attention. CXR as a LC screening tool is definitely less sensitive than chest CT [19], however it is more specific [20] and there are several clinical reasons why the cost-effectiveness of screening with chest radiograph may be worth investigating. With CXR screening there is not only a lower probability of false positive tests, but also less LC overdiagnosis and lower radiation doses than with CT screening [21, 22]. Moreover, from an economic perspective CXR is a relatively inexpensive and broadly accessible exam, two highly favorable features in a screening tool. Despite this, we are only aware of one cost-effectiveness analysis of CXR screening, based on the model developed by Caro et al. [23], where the estimated cost per discounted life-years gained ranged from $\$ 19,874$ (USD) to $\$ 59,621$ (USD). Before that, Strauss [24] made only a "crude analysis" (p. 768), leading to an estimate of $\$ 7900$ per life-year saved. It was previously reported that a population-based CXR screening program which targeted smokers (PREDICA) decreased LC mortality by $18 \%$ [9], and we made an initial estimate of the total incremental cost of screening [25]. In this paper we use the data from the PREDICA cohort [26] to investigate the cost-utility of CXR screening for LC. Our aim is to evaluate the cost-utility of the screening program in the total invitation-to-screen PREDICA cohort, including self-selected participants as well as individuals who were invited to the screening but decided not to participate. With this "intention-to-screen approach", the analysis of cost-effectiveness overcomes the well-known bias of participants' self-selection [27] and allows estimates of the screening program's overall impact on the target population.

\section{Methods}

\section{Overview of the PREDICA study}

In 1997 we began a prospective observational study, the PREDICA study (ISRCTN90639073), in a clearly defined population-based cohort of smokers. The cohort consisted of all smokers of $>10$ pack-years, aged $45-75$ years, resident in 50 communities of the Province of VareseItaly, who were screening-eligible ( $\mathrm{n}=5815$ subjects). These subjects were invited by their National Health Service (NHS) general practitioner to receive an annual LC screening with CXR for 4 years, according to an established protocol [26]. The PREDICA cohort was observed from July 1, 1997 until the end of the study (December $31,2010)$, a total of 13.5 years. The cohort's demographic characteristics, adherence to screening, LC detection results, LC survival rate, and LC mortality have been published $[9,26]$. Because some information relevant to the economic analysis was missing after 2006, the observational interval used in this study ends with December 31 , 2006. For the period until the end of 2006, clinical data were gathered, which allow to assign each patient to one of the LC stages associated with a specific value of the Quality of Life, presented later under "Utilities". Moreover, the main events related to substantial resource use were also recorded, including surgery, radiotherapy, chemotherapy, follow-up exams.

\section{Defining the invitation-to-screening group and screening participants}

In the present study of cost-effectiveness of CXR screening, the PREDICA cohort of 5,815 subjects is referred to as the Invitation-to-screening Group. Of the subjects invited to the screening program, 1244 (21\%) agreed to participate and are referred to as the Screening Participants.

\section{Defining lung cancer patient/lung cancer death}

In the Invitation-to-screening Group we defined LC patients as those who presented with a new clinical or pathological diagnosis of LC-after excluding screening-ineligible candidates (i.e., subjects unfit for surgery or with diagnosed or suspected LC as of July 1997). Through linkage with the Varese Mortality Registry and the Lombardy Health Registry of residents we identified LC deaths (codes 162.2-162.9 of the International Classification of Diseases, Edition IX) that occurred in the Invitation-to-screening Group during the study period. Information on date and cause of death were obtained from the Registry death certificates. Cause of death was definitively attributed to LC after review by the mortality review committee, as previously described [26]. The characteristics of the LC cases reported for the Screening Participants, the Invitation-to-screening Group, and the Control Group are summarized in Table 1.

\section{Timeline of economic evaluation of screening and follow-up of lung cancer cases}

For the purposes of the economic analysis, the LC cases were followed until death or December 31, 2006. For 
Table 1 Characteristics of lung cancer (LC) cases diagnosed between July 1, 1997 and December 31,2006 in screening participants, invitation-to-screening group and control group [26]

\begin{tabular}{|c|c|c|c|}
\hline Variable & $\begin{array}{l}\text { Screening participants } \\
\text { (67 LC cases) }\end{array}$ & $\begin{array}{l}\text { Invitation-to-screening } \\
\text { group ( } 245 \text { LC cases) }\end{array}$ & $\begin{array}{l}\text { Control group } \\
\text { (156 LC cases) }\end{array}$ \\
\hline Age, median (IQR) & $66(60-72)$ & $68(60-73)$ & $68(62-73)$ \\
\hline Gender M/F & $60 / 7$ & $221 / 24$ & $144 / 12$ \\
\hline \multicolumn{4}{|l|}{ Smoking habit, n (\%) } \\
\hline Ex smokers & $16(24)$ & $60(25)$ & $77(49)$ \\
\hline Smokers & $50(76)$ & $180(75)$ & $79(51)$ \\
\hline \multicolumn{4}{|l|}{ LC stage at diagnosis, n (\%) } \\
\hline 1 & $21(32)$ & $49(21)$ & $23(15)$ \\
\hline$\|$ & $3(5)$ & $13(6)$ & $9(6)$ \\
\hline III/IV & $41(63)$ & $166(73)$ & $122(79)$ \\
\hline Indeterminate/not available & 2 & 17 & 2 \\
\hline LC cases with censored follow-up*, n (\%) & $21(31 \%)$ & $57(23 \%)$ & $12(8 \%)$ \\
\hline LC follow-up months*, median (IQR) & $14.03(30.82)$ & $11.89(22.54)$ & $8.20(15.82)$ \\
\hline
\end{tabular}

IQR interquartile range, $\mathrm{Cl}$ confidence interval

* Expressed as months from LC diagnosis until death, or until the end of observational period (December 31, 2006)

${ }^{\text {a }}$ Not available in 1 screening participant

b Not available in 5 subjects of invitation-to-screening group

patients alive at the end of the observational period, we have censored observations. The different proportions of these cases in the different groups (see Table 1)-due partly to the different recruitment periods-implies a potential bias in the analysis from underestimation of both costs and QALYs for these patients. The way in which we addressed this potential source of bias is discussed below under "Utilities" and "Costs".

\section{Control group}

A Control Group consisting of uninvited and unscreened LC patients, prospectively followed-up long term, was identified and used as comparator for LC cases found in the Invitation-to-screening Group. We accessed the database of all LC cases $(n=243)$ diagnosed during calendar year 2000 among the 350,000 residents of the Varese district. The year $2000 \mathrm{LC}$ cases were chosen because (1) they were nearly contemporary with the LC cases of the Invitation-to-screening Group, (2) they came from the same geographical area, and (3) their demographic and clinico-pathological data were available and published [28]. Of the $243 \mathrm{LC}$ patients diagnosed in 2000, 156 subjects met the screening criteria as of July 1997 (birth-year cohort, smoking history, residence in the district of the cohort, uninvited to screening and unscreened) and constituted the Control Group. Follow-up of these 156 LC cases was obtained through linkage with the Varese Epidemiology Observatory [29].

\section{Management of lung cancer patients}

The LC cases diagnosed in the Invitation-to-screening Group-regardless of the detection modality by screening or outside screening - and the LC cases diagnosed in the Control Group both received NHS's usual care and treatment, with management centralized in the Varese University Hospital. The diagnostic procedures for follow-up and diagnosis of individuals with suspect LC have been described previously [26]. For patients who refused biopsy or treatment and for candidates for supportive care only, the LC diagnosis was clinico-radiologic. Treatment of ascertained LC cases-surgery, chemotherapy (induction and/or adjuvant), radiotherapy, palliationwas effected following international criteria [28], based on histologic subtype (non-small cell lung cancer, small cell lung cancer) and stage (6th ed. of TNM Classification of Malignant Tumors) [30].

\section{Economic evaluation}

We assessed LC screening program cost-utility using the public health system perspective: utility was measured through QALYs. We estimated the incremental cost-utility of LC screening through two comparisons. The obvious comparison was between screening participants and the control group; the other comparison was between the Invitation-to-screening group and the control group. The rationale for the latter comparison was to avert the self-selection bias of participants [27] and to explore the 
overall impact of invitation to screening (greater attention to early symptoms of LC among all invitees and among the general practitioners who accepted to recruit smokers for the PREDICA study).

\section{Utilities}

To assign utilities, for each patient we considered the duration of the following phases of LC clinical course: diagnosis, chemotherapy, post-operative, free of disease, progression, terminal phase. For each phase we obtained from the literature an estimate of the corresponding quality of life (QoL), specifically for non-small cell lung cancer (NSCLC) and for small cell lung cancer (SCLC). Phases and corresponding weights are reported in Table 2 for the baseline scenario [31] and for an alternative scenario based on the results of a meta-analysis [32] used for sensitivity analysis (columns 3 and 4).

A correction of QoL for false positive screens related to anxiety (QoL index $=0.88$ for 3 months) was made, according to Earle et al. [33].

After reviewing the literature on LC screening with CT, Black et al. could not draw any conclusions on whether the intervention was cost-effective [34]; it had been observed that the variety of modelling assumptions made in different economic evaluations is the main reason for the widely divergent estimates of cost-effectiveness of CT screening for LC [35]. In the present study we relied on modelling as little as possible, utilizing instead the wealth of data gathered from long-term observation of the Invitation-to-screening Group. In particular, to correct for potential bias from the varying proportions of censored survivors in the different groups, while bearing in mind the issue of ambiguity related to modelling assumptions that Black et al. [34] highlight, we used information from non-censored individuals to correct the value of utility assigned to censored observations. In this process we assigned to each of these survivors a number of QALYs equal to the median of a subset of cases with the longest follow-up (those recruited in 1997 and 1998 in the PREDICA study), conditional on the length of survival at the end of the study and whether the individual was screen-detected. The added number of QALYs was the excess, if any, between this conditional median and the actual number of QALYs through end of follow-up.

A frequent omission in LC screening economic evaluations is the lead-time bias correction [34]. In our study we assumed a lead-time bias of 9 months, based on stage distribution of screen-detected LCs in the PREDICA study [26]; then we subtracted from the utility of screendetected LC cases the minimum between 9/12 of QALY and the actual number of QALYs. This is a conservative assumption because it leads to the attribution of zero QALYs to some screen-detected patients even though they survive for a number of months. Underlying this is another conservative assumption, i.e. that the corresponding months would be spent in perfect health $(\mathrm{QoL}$ index $=1$ ). Utility values were discounted using a yearly discount rate of $3.5 \%$ [36].

\section{Costs}

Costs of LC cases were linked to three main activities: administrative work to run the screening program, screening exams, and health care services. Data on administrative costs of screening were extracted from the balance-sheet of the charity that managed the screening program; voluntary work was evaluated using marketequivalent wages. CXR screening exam costs included the cost of follow-up exams to ascertain suspicious or

Table 2 Phases of lung cancer clinical course and Quality of Life (QoL)

\begin{tabular}{|c|c|c|c|c|}
\hline \multirow{2}{*}{$\begin{array}{l}\text { Phase of clinical course } \\
\text { of lung cancer }\end{array}$} & \multicolumn{4}{|l|}{ QoL Index } \\
\hline & NSCLC (baseline) & SCLC (baseline) & $\begin{array}{l}\text { NSCLC (alternative } \\
\text { scenario) }^{\mathrm{a}}\end{array}$ & $\begin{array}{l}\text { SCLC (alternative } \\
\text { scenario) }^{\mathrm{a}}\end{array}$ \\
\hline 1. Period of diagnosis & $0.88^{d}$ & $0.95^{d}$ & $0.825^{d}$ & $0.605^{d}$ \\
\hline 2. Chemotherapy & $0.82^{\mathrm{a}}$ & $0.83^{\mathrm{a}}$ & $0.573^{c}$ & $0.353^{c}$ \\
\hline 3. Postoperative & $0.80^{b}$ & $0.80^{d}$ & $0.825^{c}$ & $0.605^{c}$ \\
\hline 4. Free of disease & $0.88^{a}$ & $0.95^{\mathrm{a}}$ & $0.825^{c}$ & $0.605^{c}$ \\
\hline 5. Disease progression & $0.69^{\mathrm{a}}$ & $0.31^{\mathrm{a}}$ & $0.573^{c}$ & $0.353^{c}$ \\
\hline 6. Terminal phase & $0.60^{\mathrm{b}}$ & $0.31^{d}$ & $0.573^{c}$ & $0.353^{c}$ \\
\hline
\end{tabular}

NSCLC non-small cell lung cancer, SCLC small cell lung cancer

a Alternative scenario described in "Methods"; Earle et al. [33]

b Manser et al. [31]

c Sturza [32]

d Where specific information on the QoL index could not be retrieved from the literature, we made the following assumptions: In column 2, for the "postoperative" phase we assumed the index for SCLC to be the same as for NSCLC, Line 1 is the same as line 3, In column 2, for the "terminal" phase we assume the index to be the same as for the "progression" 
false positive CXR screens and the cost of time spent for quality assurance and program supervision. For the latter, we estimated a workload equivalent to $20 \%$ of a full-time consultant physician for the duration of active screening. Unitary costs for health care treatment and followup were estimated from the corresponding 2012 regional tariffs, which are reported in Appendix (Table 5). These may differ slightly from National tariffs, although in several cases they are the same across Italy.

Because utilities were adjusted to account for censoring, the adjustment was also done for costs. We used the same approach as that described above for utilities, i.e. we assigned the median cost, conditional on survival at the end of follow-up and whether the case was screen-detected.

All values were expressed in 2012 Euros using the Italian National Institute of Statistics [37] health care price index. The discount rate used for utilities (3.5\%) was applied to costs.

\section{Cost-utility analysis}

The cost-effectiveness of a new intervention is usually assessed by comparing the Incremental cost-effectiveness ratio (ICER) to a threshold value of the maximum Willingness to Pay (WTP) per unit of effectiveness:

$$
\frac{\Delta C}{\Delta E}<\lambda,
$$

where $\Delta C$ and $\Delta E$ are the difference, respectively, in costs and effectiveness of the comparator, and $\lambda$ is the WTP. The inequality in the above equation can be equivalently expressed in linear form as:

$$
\lambda \cdot \Delta E-\Delta C>0 .
$$

The left side of the equation is the Incremental Net Monetary Benefit (INMB). The latter was proposed as an alternative to ICER [38] mainly because of the advantages it provides in statistical analysis; it is also widely used currently. The INMB measures the incremental net benefit of the new intervention in monetary units. In our case, the INMB is based on a measure of utility (QALYs) and can be calculated as a weighted average across health states of individuals:

$$
\begin{aligned}
I N M B= & p_{L C} \cdot I N M B_{L C}+p_{f p} \cdot I N M B_{f p} \\
& +\left(1-p_{L C}-p_{f p}\right) \cdot I N M B_{t n}
\end{aligned}
$$

where, $p$ denotes probability and subscripts refer to the following states: lung cancer $(L C)$, false positive $(f p)$ and true negative $(t n)$. INMB $B_{L C}$ is calculated using data on costs and utilities that are available for both the invitation-to-screening group and the control group. INMB $B_{f p}$ is estimated using data from the screening participants and it is based again on a conservative assumption, i.e. that only screening may lead to a false positive response. $I N M B_{f p}$ is a negative number that accounts for both additional costs and anxiety-related QoL implications of a false positive screen. Finally, $I N M B_{t n}$ is also a negative number because of the costs (administration and screening) assessed even if the individual remains healthy. We assume no difference in utility among the groups compared for the true negative.

In addition to the well-known advantages of INMB over Incremental Cost-Effectiveness Ratio (ICER) for dealing with sampling variance [39], the former approach also allows us to study the sensitivity of our results to the probability of diagnosed LC and false positive test. This is crucial for three reasons: (1) the results tend to be very sensitive to these probabilities; (2) the number of LC cases that we observed among participants (67) is relatively small, and (3) the statistical error for the estimated probability of diagnosed LC may be comparatively large. A sensitivity exercise on these probabilities may help assess the possibility of extending our results to contexts in which LC risk is higher or lower.

We made two estimates of the INMB. First, we compared Screening Participants vs. Control Group, then Intervention-to-screening Group vs. Control Group. We interpret these results as the cost-utility of screening-participation and invitation-to-screening, respectively.

\section{Sensitivity analysis}

For baseline analysis, we used the frequency of LC cases in Screening Participants and in Intervention-to-screening Group, after excluding 2 cases of overdiagnosis $(67 / 1244=0.054$ and $243 / 5815=0.042)$, to estimate $p_{L C}$. Similarly, $p_{f p}$ equals the ratio of the number of individuals with a false positive test to the number of participants $(190 / 1244=0.153)$. Sensitivity analysis was performed by replacing the baseline probability with the extremes of the $95 \%$ confidence interval (CI) of the estimated probability of LC. The other parameters we let vary in the sensitivity analysis were QoL weights (see Table 2) and cost levels. For costs, we investigated the impact of an arbitrary $20 \%$ increase in all costs, which may inform about the possibility of generalizing our results to other health care systems. We primarily used two values of the WTP that are often referred to in the literature: $€ 30,000 / Q A L Y$ and $€ 50,000 / Q A L Y$. A general analysis of the impact of the WTP was provided by estimating the cost-effectiveness acceptability curve (CEAC).

\section{Results}

Mean values for QALYs and costs of LC cases are shown in Table 3. Overall, the mean total cost per LC case was higher in Screening Participants $(€ 17,516)$ than in the 
Table 3 Mean costs and QALYs per lung cancer (LC) case in screening participants, invitation-to-screening group and control group

\begin{tabular}{lccc}
\hline & Screening participants & Invitation-to-screening group & Control group \\
\hline Mean costs per LC case & & & $84(0)$ \\
Screening organization and administration & $307(0)$ & $16(35)$ & - \\
CXR screening & $60(44)$ & $16,067(4948)$ & - \\
LC management & $17,149(4783)$ & $16,167(4729)$ & $15,503(4518)$ \\
Mean total cost per LC case & $17,516(4755)$ & $1.49(1.9)$ & $15,503(4521)$ \\
Mean QALYs (baseline scenario) & $1.70(2.1)$ & $1.07(1.7)$ \\
\hline
\end{tabular}

All amounts are in Euros 2012. Standard deviation in parenthesis. Values are rounded to the nearest integer

control group $(€ 15,503)$. The individual component of the costs related to organization and administration of the screening program (€307) was obtained by dividing the total cost by the number of participants. This component is the same for LC cases (Table 3) and true negative individuals in Screening Participants. The LC management costs were also higher in Screening Participants $(€ 17,149)$ than in the control group $(€ 15,503)$.

Looking at the comparison between the Invitation-toscreening Group and the Control Group, the incremental total cost $(€ 16,167-15,503)$ was $€ 664$. Costs related to screening activities were lower for the Invitationto-screening Group $(€ 100)$ than for screening participants. This is because the Invitation-to-screening group includes non-participants for whom screening costs were imputed to Screening Participants (see above). Finally, LC management cost was higher, on average, in the Invitation-to-screening Group $(€ 16,067)$ than in the control group $(€ 15,503)$ (Table 3$)$.

The average (discounted) number of QALYs since diagnosis was $1.70,1.49$, and 1.07 , respectively, for screening participants, the invitation-to-screening group, and the control group. Accordingly, on average Screening Participants gained 0.63 QALYs and the Invitation-to-screening Group gained 0.42 QALYs, relative to the Control Group. The combination of these results with costs and utilities of true negative and false positive individuals leads to an estimate for the individual level INMB of $€ 368$ for screening participation and $€ 365$ for invitation-to-screening, with a WTP of $€ 30,000$ per QALY (Table 4). With a WTP of $€ 50,000$ per QALY, the estimated INMB increases, respectively, to $€ 959$ for screening participation and $€ 692$ for invitation-to-screening. Table 4 also shows the probability that, taking sampling variance into account, the intervention is cost-effective for the two levels of WTP considered, under the main and the sensitivity scenarios. In particular, it is worth noting that a $20 \%$ increase in unitary costs does not significantly change the results.

The CEAC provides a more general picture of the relationship between the level of WTP and the probability that screening is cost-effective. Figures 1 and 2 show the CEAC for the baseline and sensitivity scenarios. Referring once more to the values of WTP per QALY of $€ 30,000$ and $€ 50,000$, the probability that screening participation is cost-effective is, respectively, 0.79 and 0.9 in the baseline scenario. The corresponding probabilities for invitation-to-screening are 0.95 and 0.97 . Table 4 summarizes the results of the sensitivity analysis.

\section{Discussion}

Based on long-term observation of the population-based PREDICA study of CXR screening in smokers [26], our assessment of cost-effectiveness of CXR screening for LC indicates an expected INMB (WTP: $€ 30,000$ per QALY) of $€ 368$ for the comparison between Screening Participants and Control Group in the baseline analysis. This finding implies a 0.79 probability that screening participation is cost-effective. Notably, in addition to screening-related costs, participants showed higher LC management costs due to more frequent surgical procedures performed as a result of early LC detection. Moreover, compared with the Control Group, the Screening Participants had markedly improved 5-year survival (30.5 vs. $13.0 \%$ ) [26] and longer median follow-up (Table 1), which added cost to LC management. Because we observed only 67 LC cases among Screening Participants $(\mathrm{n}=1244)$, despite the large number of individuals ( $\mathrm{n}=5815)$ invited to screening, the variance around the central estimate of the INMB is wide. This confirms that the assessment of a screening program cost-effectiveness requires substantial investment of resources and prolonged observation.

Our study has limitations. First, it is not a randomized study and therefore is subject to the selection bias of screening volunteers, as previously noted [27]. Second, in our study the proportion of censored observations was different across groups. We addressed this potential bias by adjusting utilities and costs of censored observations using the wealth of information available on non-censored patients. 
Table 4 Sensitivity analysis testing the probability that screening participation or invitation-to-screening is cost-effective

\begin{tabular}{|c|c|c|c|c|}
\hline & \multicolumn{2}{|l|}{ INMB } & \multicolumn{2}{|l|}{ Probability cost-effective } \\
\hline & WTP (€ per QALY) 30,000 & WTP (€ per QALY) 50,000 & WTP ( $€$ per QALY) 30,000 & WTP (€ per QALY) 50,000 \\
\hline \multicolumn{5}{|c|}{ Screening participation (group P vs. group C) } \\
\hline Base-case & 368 & 959 & 0.79 & 0.89 \\
\hline High prob of $L C\left(p_{L C}=6.79 \%\right)$ & 609 & 1376 & 0.86 & 0.92 \\
\hline Low prob of $L C\left(p_{L C}=4.2 \%\right)$ & 160 & 600 & 0.67 & 0.84 \\
\hline Alternative QoL & 134 & 568 & 0.63 & 0.80 \\
\hline Higher costs & 265 & 856 & 0.72 & 0.87 \\
\hline \multicolumn{5}{|c|}{ Invitation-to-screening (group I vs. group C) } \\
\hline Base-case & 365 & 692 & 0.95 & 0.97 \\
\hline High prob of $L C\left(p_{L C}=4.73 \%\right)$ & 438 & 816 & 0.96 & 0.97 \\
\hline Low prob of LC $\left(p_{L C}=3.68 \%\right)$ & 311 & 601 & 0.94 & 0.97 \\
\hline Alternative QoL & 260 & 519 & 0.90 & 0.93 \\
\hline Higher costs & 339 & 667 & 0.94 & 0.96 \\
\hline
\end{tabular}

INMB incremental net monetary benefit, WTP willingness to pay, LC lung cancer

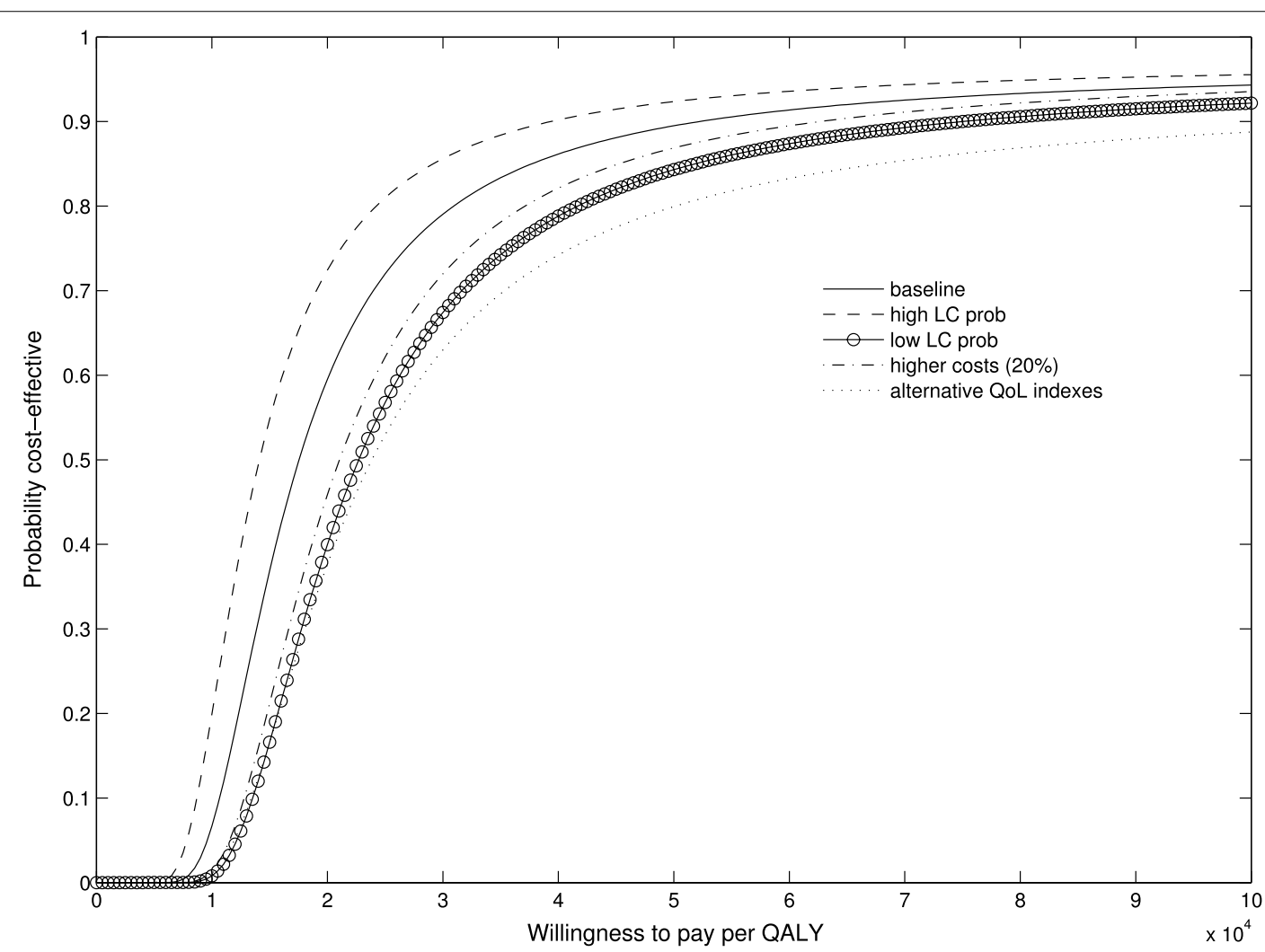

Fig. 1 Cost-effectiveness acceptability curve for screening participation (screening participants vs. control group) in the baseline and sensitivity scenarios (see text, "Sensitivity analysis")

A strength of our study is the Control Group consisting of uninvited and unscreened LC patients, with $92 \%$ complete follow-up. The Control Group LCs were nearly contemporary to those of the Invitation-to-screening
Group and were gathered from the same geographical area. Another strength of the study is that information was gathered on LC cases that occurred in individuals who were invited to the screening program and decided 


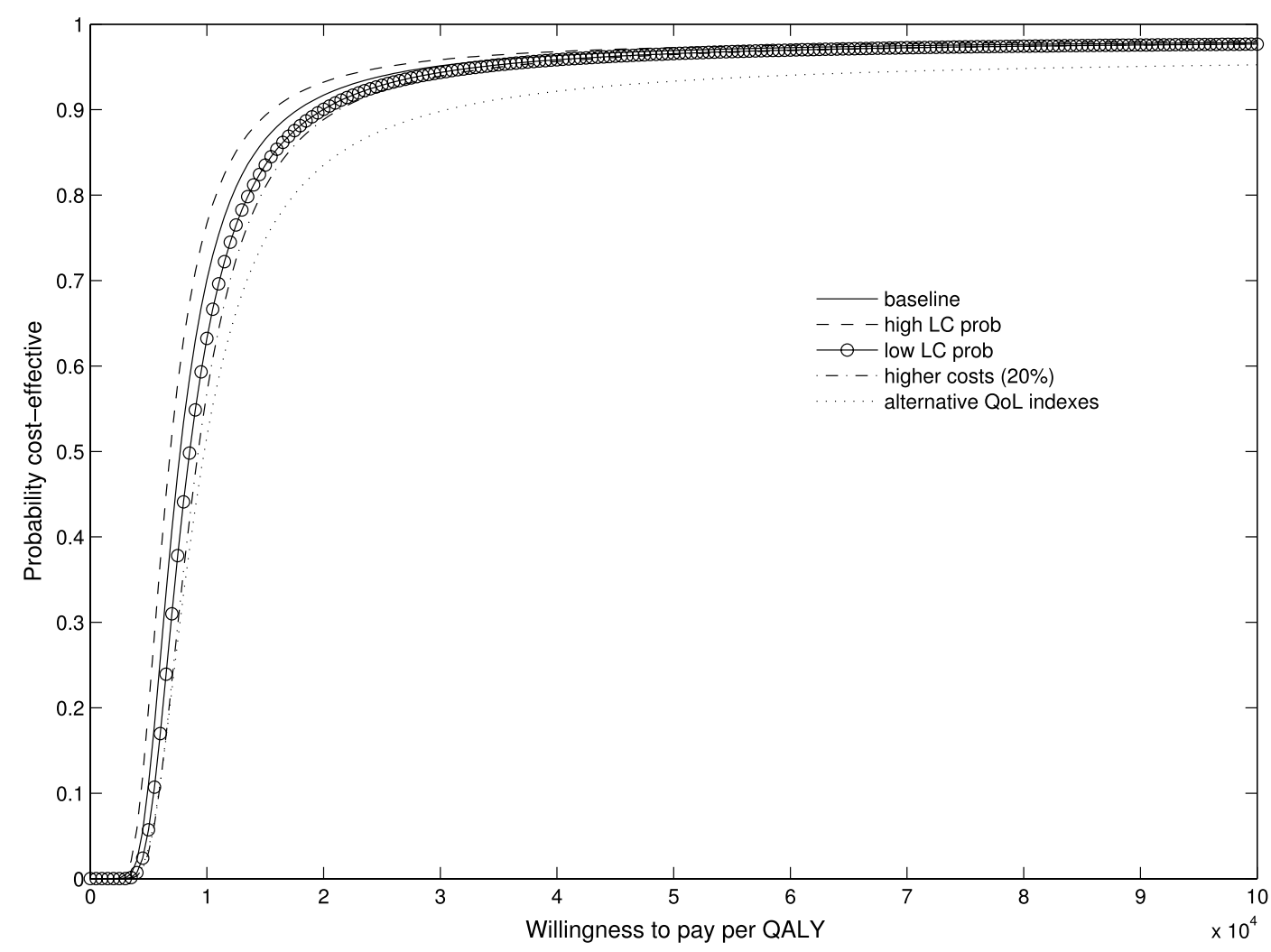

Fig. 2 Cost-effectiveness acceptability curve for invitation-to-screening (invitation-to-screening group vs. control group) in the baseline and sensitivity scenarios (see text, "Sensitivity analysis")

not to participate. Thus, we were able to assess the overall cost-utility of invitation-to-screening by comparing the Invitation-to-screening Group with the Control Group. This comparison yielded an expected value of individual INMB of $€ 365$, indicating that invitation-to-screening is cost-effective with a probability of 0.95 . The small difference with the central estimate of INMB obtained from the previous comparison ( $€ 368$ vs. $€ 365)$ is due to the fact that invited subjects who did not participate had LC costs similar to the Control Group, but achieved more QALYs.

Clearly, our estimates may be affected by local context (costs, incidence of LC, WTP); however, our probabilistic sensitivity analysis showed limited impact of changes in these variables on results.

The estimated probability that the intervention is costeffective is higher for invitation-to-screening than for screening participation, despite the very close central estimates for the INMB. This finding is related to the variance of the sample mean, which is greater when Screening Participants (a subset of the invitation-to-screening group) are compared with the Control Group (67 LC cases vs. 156). Moreover, screening invitation per se, regardless of participation, might have an impact on $\mathrm{LC}$ management. The impact of invitation and its economic implications are potentially of great interest and worth exploring further in different contexts. There may also be relevant policy implications. Because the number of invited individuals typically exceeds the number of participants in screening programs, the total net benefit of the program (individual INMB times the population size) should also take into account any impact on the invited population, in addition to the effect on participants.

Results from the PREDICA study suggest that the total net benefits of a LC screening program may be greater than expected based on the analysis of screened individuals only. Implementation of the PREDICA annual CXR screening program at population level in smokers of the Varese area was associated with an $18 \%$ reduction of LC-specific mortality [9]. Because our study shows that CXR screening of high-risk smokers can be "good value for the money", the findings may help decisionmakers allocate more efficiently the available health care resources. The NLST trial showed that screening for LC with CT reduced LC mortality by $20 \%$ compared with CXR screening [11]. Based on this finding, leading cancer organizations currently recommend LC screening with LC low-dose CT [12, 40]. However, low-dose CT screening is an expensive technique not readily available in 
many parts of the world. Moreover, low-dose CT screening is associated with a relevant rate of overdiagnosis and of false positive findings [11, 41]. It has been emphasized that if early detection of LC provides a benefit, it does so regardless of whether the detection is made by $\mathrm{CT}$ or CXR screening; however, the feasibility of the detection method applied to a large number of subjects as a public health measure is essential [42]. Chest radiography is a simple exam with modest radiation exposure. It is widely available, relatively inexpensive, and non-invasive. Additionally, CXR screening causes negligible overdiagnosis of LC [21] and a low rate of false-positive results [22, 26].

In conclusion, based on the PREDICA study $[9,26]$, annual CXR screening of high-risk smokers in the general practice setting is a feasible and affordable intervention that has high probability of being cost-effective. This finding may have important policy implications in terms of resource allocation in health care systems. However, before recommending annual screening of heavy smokers by CXR, the results obtained in the present study need to be validated by other similar studies in different geographical areas and different health systems.

\begin{abstract}
Abbreviations
CEAC: cost-effectiveness acceptability curve; CT: computed tomography; CXR: chest X-ray; fp: false positive; ICER: incremental cost-effectiveness ratio; INMB: incremental net monetary benefit; LC: lung cancer; NHS: National Health System; NLST: National Lung Screening Trial; NSCLC: non-small cell lung cancer; QALY: quality-adjusted life year; QoL: Quality of Life; tn: true negative; WTP: willingness to pay.
\end{abstract}

\section{Authors' contributions}

PP, AP, LD, Al were responsible for study development and wrote the report. NR, EN, MC, MP, WM carried out data entry and protection of data and quality assurance monitoring. PP, Al, AP performed statistical analysis and interpretation of data. All authors read and approved the final manuscript.

\section{Author details}

${ }^{1}$ Department of Economics, University of Verona, Via dell'Artigliere 19, 37129 Verona, Italy. ${ }^{2}$ Department of Public Health and Community Medicine, University of Verona, Verona, Italy. ${ }^{3}$ Center for Thoracic Surgery, University of Insubria, Ospedale di Circolo, Varese, Italy. ${ }^{4}$ Department of Radiology,
Ospedale S. Antonio Abate, Gallarate, Italy. ${ }^{5}$ Department of Prevention, Public Health Trust, Trento, Italy.

\section{Acknowledgements}

We gratefully acknowledge the contribution of the Residents of the Center for Thoracic Surgery, University of Insubria, Varese in the years 2013-2014 for their assistance in carrying out the evaluation of the LC screening program.

\section{Compliance with ethical guidelines}

\section{Competing interests}

The authors declare that they have no competing interests.

\section{Consent statement}

Consent for publication of the raw data in this study was not obtained but the dataset is fully anonymous in a manner that can easily be verified by the dataset user.

\section{Data availability}

The dataset for the economic evaluation of the health intervention in this study is fully anonymous and can be obtained, upon motivated request, from the corresponding author.

\section{Ethics}

Signed informed consent was obtained from all individuals who accepted to participate in screening. For nonparticipants, informed consent was waived and their names were encrypted, as approved by the Varese Hospital and Health District Ethics Committee. Approval of this study and permission to access and analyze the anonymized linked data reported in this article was granted by the Varese Hospital and Health District Ethics Committee (May 20, 1997). The observational screening study which forms the basis of this economic evaluation is registered (ISRCTN90639073).

\section{Funding}

This study was financed with funds granted to LD by the following bodies: University of Insubria Research Funds FAR2013-FAR2014; Lions Club Varese Host annual grant 2014; Associazione PREDICA Onlus, Varese in the years 2013-2014. The authors declare that these funding bodies had no role in the study design, in the collection, analysis, and interpretation of data, in the writing of the manuscript, and in the decision to submit the manuscript for publication.

\section{Standards of reporting}

The standards of reporting are in accordance with STROBE recommendations for observational studies, and with CHEERS recommendations for reporting economic evaluations of health interventions.

\section{Appendix}

See Table 5. 
Table 5 Cost of health care services for diagnosis, staging, treatment and follow-up of lung cancer

\begin{tabular}{|c|c|c|}
\hline Cost components & Unitary cost (EURO 2012) & Source \\
\hline \multicolumn{3}{|l|}{ Cyto-/histologic confirmation of NSCLC/SCLC } \\
\hline Bronchoscopy with biopsy & 184.74 & Tariff 2012 BRL \\
\hline Cytology of sputum/pleural fluid & 27.45 & Tariff 2012 BRL \\
\hline CT-guided fine needle aspirate and cytology & 160.99 & Tariff 2012 BRL \\
\hline Day hospital stay & 624.00 & Tariff 2012 BRL \\
\hline Total & 997.18 & \\
\hline \multicolumn{3}{|l|}{ Evaluation of stage I-IV LC } \\
\hline Blood cells count, chemistry, markers, gases & 117.25 & Tariff 2012 BRL \\
\hline EKG & 11.60 & Tariff 2012 BRL \\
\hline CXR exam, dual projection & 17.40 & Tariff 2012 BRL \\
\hline Chest $\mathrm{CT}$ without and with contrast & 164.67 & Tariff 2012 BRL \\
\hline Abdomen $\mathrm{CT}$ without and with contrast & 168.37 & Tariff 2012 BRL \\
\hline Head CT without and with contrast & 159.93 & Tariff 2012 BRL \\
\hline Bone scintigraphy & 111.90 & Tariff 2012 BRL \\
\hline Spirometry & 23.75 & Tariff 2012 BRL \\
\hline Hospital stay (average 7 days) & 4160.00 & Tariff 2012 BRL \\
\hline Total & 4934.87 & \\
\hline \multicolumn{3}{|l|}{ Evaluation of indeterminate stage LC } \\
\hline Blood cells count, chemistry, markers, gases & 117.25 & Tariff 2012 BRL \\
\hline EKG & 11.60 & Tariff 2012 BRL \\
\hline CXR exam, dual projection & 17.40 & Tariff 2012 BRL \\
\hline Chest $\mathrm{CT}$ without and with contrast & 164.67 & Tariff 2012 BRL \\
\hline Abdomen $\mathrm{CT}$ without and with contrast & 168.37 & Tariff 2012 BRL \\
\hline Head CT without and with contrast & 159.93 & Tariff 2012 BRL \\
\hline Hospital stay (average 4 days) & 2496.00 & Tariff 2012 BRL \\
\hline Total & 3135.22 & \\
\hline Operating room occupancy (average 180 min.) & 3000.00 & Varese Hospital Admin. \\
\hline Operating room materials & 1500.00 & Varese Hospital Admin. \\
\hline Hospital stay in surgical unit (average 12 days) & 6600.00 & Varese Hospital Admin. \\
\hline Total & $11,100.00$ & \\
\hline \multicolumn{3}{|l|}{ Chemotherapy for LC stage III } \\
\hline 6 Cycles (Cisplatin + Gemcitabine) & 8989.62 & Oncology Unit Varese Hospital \\
\hline \multicolumn{3}{|l|}{ Chemotherapy for LC stage IV } \\
\hline 3 Cycles (Cisplatin + Gemcitabine) & 4494.81 & Oncology Unit Varese Hospital \\
\hline Palliative radiotherapy & 4934.55 & Radiotherapy Unit Varese Hosp. \\
\hline Radical radiotherapy (50-60 Gy) & 7849.46 & Radiotherapy Unit Varese Hosp \\
\hline Chemo-Radiotherapy combined & $10,091.03$ & Onc./Radiot.Units Varese Hosp. \\
\hline Supportive/palliative therapy & 2759.76 & Tariff 2012 BRL \\
\hline Terminal phase care (1 month) & 1800.00 & Tariff 2012 BRL \\
\hline \multicolumn{3}{|l|}{ Follow-up: first year } \\
\hline 3 Physical exams & 74.10 & Tariff 2012 BRL \\
\hline 2 CXR exams, dual projection & 34.80 & Tariff 2012 BRL \\
\hline 2 Blood cells counts, chemistry, markers, gases & 234.50 & Tariff 2012 BRL \\
\hline 1 Chest $\mathrm{CT}$ exam without and with contrast & 164.67 & Tariff 2012 BRL \\
\hline Total & 508.07 & \\
\hline \multicolumn{3}{|l|}{ Follow-up: second year } \\
\hline 2 Physical exams & 49.40 & Tariff 2012 BRL \\
\hline 1 CXR exam, dual projection & 17.40 & Tariff 2012 BRL \\
\hline 2 Blood cells counts, chemistry, markers, gases & 234.50 & Tariff 2012 BRL \\
\hline
\end{tabular}


Table 5 continued

\begin{tabular}{lcc}
\hline Cost components & Unitary cost (EURO 2012) & Source \\
\hline 1 Chest CT exam without and with contrast & 164.67 & Tariff 2012 BRL \\
Total & 465.97 \\
Follow-up: third and subsequent years & \\
2 Physical exams & 49.40 \\
1 Blood cells count, chemistry, markers, gases & 117.25 \\
1 Chest CT exam without and with contrast & 164.67 & Tariff 2012 BRL \\
Total & 331.32 & Tariff 2012 BRL \\
\hline
\end{tabular}

Source of information about cost is indicated

NSCLC non-small cell lung cancer, SCLC small cell lung cancer, BRL Bulletin of Region Lombardy, CT computed tomography, CXR chest X-ray, EKG electrocardiogram, $L C$ lung cancer

Received: 15 March 2015 Accepted: 4 September 2015

Published online: 12 September 2015

\section{References}

1. Jemal A, Bray F, Center MM, Ferlay J, Ward E, Forman D. Global cancer statistics. CA Cancer J Clin. 2011;61(2):69-90.

2. Dela Cruz CS, Tanoue LT, Matthay RA. Lung Cancer: epidemiology, etiology, and prevention. Clin Chest Med. 2011;32(4):605-44.

3. Burns DM. Primary prevention, smoking, and smoking cessation: implications for future trends in lung cancer prevention. Cancer. 2000;89(11 Suppl):S2506-9.

4. Roth K, Nilsen TI, Hatlen E, Sorensen KS, Hole T, Haaverstad R. Predictors of long time survival after lung cancer surgery: a retrospective cohort study. BMC Pulm Med. 2008;8:22.

5. Wright G, Manser RL, Byrnes G, Hart D, Campbell DA. Surgery for non-small cell lung cancer: systematic review and meta-analysis of randomised controlled trials. Thorax. 2006;61(7):597-603.

6. Flehinger HJ, Melamed MR, Zaman MB, Heelan RT, Perchick W, Martini N Resectability of lung cancer and survival in the New York Lung Cancer Detection Program. World J Surg. 1981;5(5):681-7.

7. Mangone L, Minicozzi P, Vicentini M, Giacomin A, Caldarella A, Cirilli C, Falcini F, Giorgi Rossi P, Sant M. Key factors influencing lung cancer survival in northern Italy. Cancer Epidemiol. 2003;37(3):226-32.

8. Henschke Cl, Boffetta P, Gorlova O, Yip R, Delancey JO, Foy M. Assessment of lung-cancer mortality reduction from CT Screening. Lung Cancer. 2011;71(3):328-32.

9. Dominioni L, Poli A, Mantovani W, Pisani S, Rotolo N, Paolucci M, Sessa F, Conti V, D'Ambrosio V, Paddeu A, Imperatori A. Assessment of lung cancer mortality reduction after chest $X$-ray screening in smokers: a populationbased cohort study in Varese, Italy. Lung Cancer. 2013;80(1):50-4.

10. Nakayama T, Baba T, Suzuki T, Sagawa M, Kaneko M. An evaluation of chest X-ray screening for lung cancer in Gunma prefecture, Japan: a population-based case-control study. Eur J Cancer. 2002;38(10):1380-7.

11. National Lung Cancer Screening Trial Research team, Aberle DR, Adams AM, Berg CD, Black WC, Clapp JD, Fagerstrom RM, Gareen IF, Gatsonis C, Marcus PM, Sicks JD. Reduced lung-cancer mortality with low-dose computed tomographic screening. New Engl J Med. 2011;365(5):395-409.

12. Wender R, Fontham ET, Barrera E Jr, Colditz GA, Church TR, Ettinger DS, Etzioni R, Flowers CR, Gazelle GS, Kelsey DK, LaMonte SJ, Michaelson JS, Oeffinger KC, Shih YC, Sullivan DC, Travis W, Walter L, Wolf AM, Brawley OW, Smith RA. American Cancer Society Lung Cancer Screening Guidelines. CA Cancer J Clin. 2013:63(2):107-17.

13. Marshall D, Simpson KN, Earle CC, Chu CW. Economic decision analysis model of screening for lung cancer. Eur J Cancer. 2001;37(14):1759-67.

14. Mahadevia PJ, Fleisher LA, Frick KD, Eng J, Goodman SN, Powe NR. Lung cancer screening with helical computed tomography in older adult smokers: a decision and cost-effectiveness analysis. JAMA. 2003;289(3):313-22
15. Goulart BH, Bensink ME, Mummy DG, Ramsey SD. Lung cancer screening with low-dose computed tomography: costs, national expenditures, and cost-effectiveness. J Natl Compr Canc Netw. 2012;10(2):267-75.

16. Heuvers ME, Stricker BH, Aerts JG. Generalizing lung-cancer screening results. N Engl J Med. 2012;366(2):192-3.

17. Grosu HB, Eapen GA, Jimenez CA, Morice RC, Ost D. Lung cancer screening: making the transition from research to clinical practice. Curr Opin Pulm Med. 2012;18(4):295-303.

18. McMahon PM, Kong CY, Bouzan C, Weinstein MC, Cipriano LE, Tramontano AC, Johnson BE, Weeks JC, Gazelle GS. Cost-effectiveness of computed tomography screening for lung cancer in the United States. J Thorac Oncol. 2011;6(11):1841-8.

19. Henschke Cl, McCauley DI, Yankelevitz DF, Naidich DP, McGuinness G, Miettinen OS, Libby DM, Pasmantier MW, Koizumi J, Altorki NK, Smith JP. Early Lung Cancer Action Project: overall design and findings from baseline screening. Lancet. 1999;354(9173):99-105.

20. Gavelli G, Giampalma E. Sensitivity and specificity of chest X-ray screening for lung cancer: review article. Cancer. 2000;89(11 Suppl):S2453-6.

21. Strauss GM, Dominioni L. Chest X-ray screening for lung cancer: overdiagnosis, endpoints, and randomized population trials. J Surg Oncol. 2013;108(5):294-300.

22. Gossner J. Lung cancer screening-don't forget the chest radio graph. World J Radiol. 2014;6(4):116-8.

23. Caro JJ, Klittich WS, Strauss G. Could chest X-ray screening for lung cancer be cost-effective? Cancer. 2000;89(11 Suppl):S2502-5.

24. Strauss GM. Screening for lung cancer: An evidence-based synthesis. Surg Oncol Clin N Am. 1999;8(4):747-74.

25. Dominioni L, Rotolo N, Poli A, Castiglioni M, Mangini M, Spagnoletti M Paolucci M, Paddeu A, Mantovani W, Zanini A, Imperatori A. Cost of a population-based programme of chest $X$-ray screening for lung cancer. Monaldi Arch Chest Dis. 2013;79(2):67-72.

26. Dominioni L, Rotolo N, Mantovani W, Poli A, Pisani S, Conti V, Paolucci M, Sessa F, Paddeu A, D’Ambrosio V, Imperatori A. A population-based cohort study of chest $X$-ray screening in smokers: lung cancer detection findings and follow-up. BMC Cancer. 2012;17:12-8.

27. Dominioni L, Rotolo N, Poli A, Paolucci M, Sessa F, D'Ambrosio V, Paddeu A, Mantovani W, Imperatori A. Self-selection effects in smokers attending lung cancer screening. A 9.5-year population-based cohort study in Varese, Italy. J Thorac Oncol. 2010;5(4):428-35.

28. Imperatori A, Harrison RN, Leitch DN, Rovera F, Lepore G, Dionigi G, Sutton P, Dominioni L. Lung cancer in Teesside (UK) and Varese (Italy): a comparison of management and survival. Thorax. 2006;61(3):232-9.

29. Azienda Sanitaria Locale della Provincia di Varese. Varese Province Epidemiology Observatory. Available at: http://www.asl.varese.it/. Accessed 23 May 2014

30. Mountain CF. Revisions in the international system for staging lung cancer. Chest. 1997;111(6):1710-7.

31. Manser R, Dalton A, Carter R, Byrnes G, Elwood M, Campbell DA. Costeffectiveness analysis of screening for lung cancer with low dose spiral $\mathrm{CT}$ (computed tomography) in the Australian setting. Lung Cancer. 2005:48(2):171-85. 
32. Sturza J. A review and meta-analysis of utility values for lung cancer. Med Decis Making. 2010;30(6):685-93.

33. Earle CC, Chapman RH, Baker CS, Bell CM, Stone PW, Sandberg EA, Neumann PJ. Systematic overview of cost-utility assessments in oncology. J Clin Oncol. 2000;18(18):3302-17.

34. Black C, Bagust A, Boland A, Walker S, McLeod C, De Verteuil R, Ayres J, Bain L, Thomas S, Godden D, Waugh N. The clinical effectiveness and cost-effectiveness of computed tomography screening for lung cancer: systematic reviews. Health Technol Assess. 2006;10(3):1-90.

35. Marshall HM, Bowman RV, Yang IA, Fong KM, Berg CD. Screening for lung cancer with low-dose computed tomography: a review of current status. J Thorac Dis. 2013;5(Suppl 5):S524-39.

36. National Institute for Health and Clinical Excellence Guide to the Methods of Technology Appraisal. London: National Institute for Health and Clinical Excellence 2008

37. Istituto Nazionale di Statistica (ISTAT). Available at: http://rivaluta.istat.it. Accessed November 25, 2013.

38. Stinnett AA, Mullahy J. Net health benefits a new framework for the analysis of uncertainty in cost-effectiveness analysis. Med Decis Making 1998;18(Suppl2):S68-80
39. Briggs AH, O'Brien BJ, Blackhouse G. Thinking outside the box: recent advances in the analysis and presentation of uncertainty in cost-effectiveness studies. Ann Rev Public Health. 2002;23(1):377-401.

40. Jaklitsch MT, Jacobson FL, Austin JHM, Field JK, Jett JR, Keshavjee S, MacMahon H, Mulshine JL, Munden RF, Salgia R, Strauss GM, Swanson SJ, Travis WD, Surgarbaker DJ. The American Association for Thoracic Surgery guidelines for lung cancer screening using low-dose computed tomography scans for lung cancer survivors and other high-risk groups. J Thorac Cardiovasc Surg. 2012;144(1):33-8.

41. Patz EF, Pinsky P, Gatsonis C, Sicks JD, Kramer BS, Tammemagi MC, Chiles C, Black WC, Aberle DR, Overdiagnosis Manuscript Writing Team NLST. Overdiagnosis in low-dose computed tomography screening for lung cancer. JAMA Intern Med. 2014;174(2):269-74.

42. Kussman RS. The overdiagnosis theory in lung cancer screening: does it make any sense? J Surg Oncol. 2013;109(3):177-8.

\section{Submit your next manuscript to BioMed Central and take full advantage of:}

- Convenient online submission

- Thorough peer review

- No space constraints or color figure charges

- Immediate publication on acceptance

- Inclusion in PubMed, CAS, Scopus and Google Scholar

- Research which is freely available for redistribution

Submit your manuscript at

www.biomedcentral.com/submit

C Biomed Central 